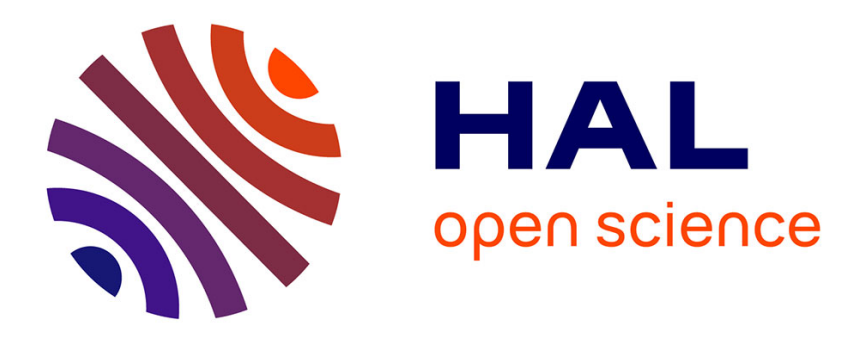

\title{
Fatigue photostructurale dans les films de sélénium amorphe
}

C. Viger, G. Fleury, C. Vautier

\section{To cite this version:}

C. Viger, G. Fleury, C. Vautier. Fatigue photostructurale dans les films de sélénium amorphe. Journal de Physique Lettres, 1981, 42 (22), pp.495-499. 10.1051/jphyslet:019810042022049500 . jpa00231985

\section{HAL Id: jpa-00231985 https://hal.science/jpa-00231985}

Submitted on 1 Jan 1981

HAL is a multi-disciplinary open access archive for the deposit and dissemination of scientific research documents, whether they are published or not. The documents may come from teaching and research institutions in France or abroad, or from public or private research centers.
L'archive ouverte pluridisciplinaire HAL, est destinée au dépôt et à la diffusion de documents scientifiques de niveau recherche, publiés ou non, émanant des établissements d'enseignement et de recherche français ou étrangers, des laboratoires publics ou privés. 


\title{
Fatigue photostructurale dans les films de sélénium amorphe (*)
}

\author{
C. Viger, G. Fleury et C. Vautier \\ Laboratoire d'Etude des Couches Minces Amorphes et Polycristallines, Faculté des Sciences de Rouen, \\ F 76130 Mont-Saint-Aignan, France
}

(Reşu le 3 juillet 1981, accepté le 2 octobre 1981)

\begin{abstract}
Résumé. - L'étude in situ de la fatigue photostructurale sur des couches de Se amorphe préparées par évaporation " flash » souligne l'importance du modèle des défauts chargés (M.D.S.). L'influence de la température sur la redistribution au hasard des défauts et la réversibilité des effets photostructuraux est envisagée. Une explication qualitative de l'extinction optique peut être donnée.
\end{abstract}

\begin{abstract}
Photostructural fatigue in amorphous selenium films, prepared by flash evaporation technique and studied in situ, emphasizes the interest of charged defects model (M.D.S.). The role of temperature on random distribution of defects and photostructural reversibility is considered. A qualitative explanation of optical quenching can be given.
\end{abstract}

1. Introduction. - Le modèle des défauts chargés dans les chalcogènes $[1,2]$ fait apparaître le rôle fondamental des liaisons pendantes. Un certain nombre de phénomènes expérimentaux : fatigue photostructurale, saturation à basse température, extinction optique trouvent une interprétation dans la modification de la répartition spatiale des défauts dans le matériau. La contribution de la température de transition vitreuse à la répartition des défauts conduit à la notion de réversibilité et de reproductibilité des phénomènes.

2. Méthodes expérimentales. - Les couches de sélénium amorphe, en structure planaire, d'épaisseur $1 \mu \mathrm{m}$, sont préparées par évaporation flash dans un vide de $10^{-7}$ mbar sur un substrat de V.I.R.3 maintenu à $293 \mathrm{~K}$ et sont étudiées in situ. Un cryostat permet de réguler la température des couches entre $73 \mathrm{~K}$ et $473 \mathrm{~K}$ à $0,5 \mathrm{~K}$ près. L'éclairement monochromatique est obtenu à partir d'un monochromateur à réseau (2 000 traits $/ \mathrm{mm}$ ) éclairé par une source quartz halogène dont le flux lumineux est asservi par une photodiode. Les mesures de photocourant sont effectuées sous un champ de $5 \times 10^{5} \mathrm{~V} \cdot \mathrm{m}^{-1}$ correspondant à un régime ohmique.

(*) Cet article a été présenté au Congrès de la Société Française de Physique (Clermont-Ferrand, juillet 1981) dans le cadre du Colloque « Verres et Amorphes ».

This article was presented at the Meeting of the French Physical Society (Clermont-Ferrand, July 1981) at the Symposium on "Glass and Amorphous Materials ".
3. Modèle de défauts chargés et photoconduction. Le modèle des défauts chargés peut être résumé par le schéma de bande de Mott, Davis, Street résumé sur la figure 1 .

- Les centres $\mathrm{D}^{0}$ sont des centres neutres possédant un électron de « lone pair " non apparié.

- Les centres $\mathrm{D}^{-}$sont des centres une fois coordinés contenant deux paires d'électrons en position de « lone pair ».

- Les centres $\mathrm{D}^{+}$sont des centres trois fois coordinés sans électron de « lone pair».

Pour des températures inférieures à $T_{\mathrm{g}}$ les défauts sont "gelés " et la densité des centres $\mathrm{D}^{0}\left(N_{0}\right)$ est négligeable par rapport à la densité totale des défauts

$$
N_{\mathrm{D}}=N \cdot \exp \left(-\frac{U_{\mathrm{LP}}}{2 k T_{\mathrm{g}}}\right)
$$

avec $U_{L P}$ énergie de formation de "lone pair".

Sous l'influence d'une excitation optique, les porteurs libres créés sont rapidement capturés par les centres $\mathrm{D}^{+}, \mathrm{D}^{-}$suivant les réactions

$$
\begin{array}{ll}
D^{+}+e_{b c} \rightarrow D^{0} & \text { (capture d'un électron libre) } \\
D^{-} \rightarrow D^{0}+e_{b v} & \text { (capture d'un trou libre) }
\end{array}
$$

ce qui accroît très sensiblement la densité des centres $\mathrm{D}^{0}\left(\Delta N_{0}\right)$. Deux situations sont alors possibles : 

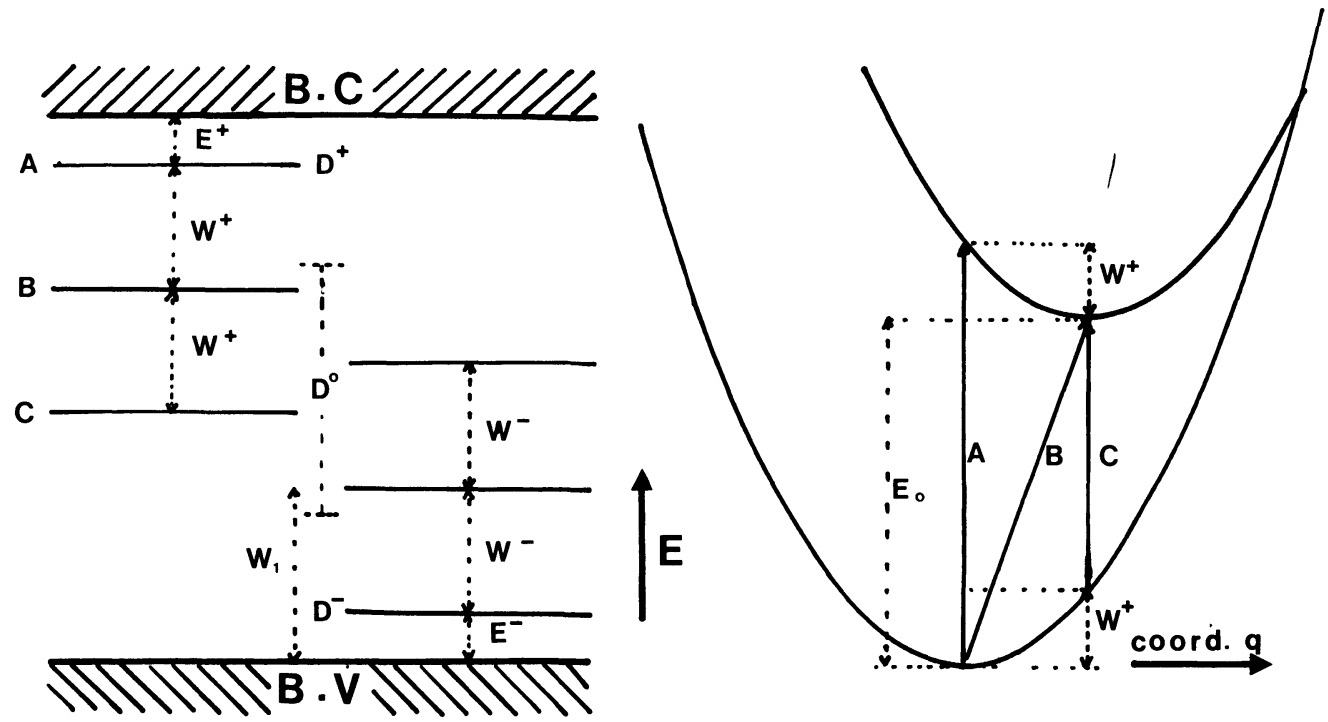

Fig. 1. - Modèle de bande pour les transitions sur les défauts des chalcogènes de Street et Mott.

[Band model for transitions on Street and Mott chalcogenides defects.]

- réexcitation thermique dans les bandes respectives, par exemple génération d'un trou libre responsable de la photoconduction

$$
D^{0}+e_{b v} \rightarrow D^{-}
$$

- échange d'électrons entre deux centres $\mathrm{D}^{0}$

$$
2 \mathrm{D}^{0} \rightarrow \mathrm{D}^{+}+\mathrm{D}^{-}
$$

c'est-à-dire recombinaison des centres $\mathrm{D}^{0}$.

Comme dans la théorie de Simmons et Taylor [3], ce sont les mêmes centres qui jouent le rôle de pièges ou de centres recombinants, en conséquence, seul l'état énergétique des centres (dû à une relaxation du matériau en fonction de la charge et des liaisons) est différent. En général, la recombinaison est non radiative [4] et l'énergie est dissipée sous forme de phonons.

La photoconduction est proportionnelle au nombre de centre $D^{0}$ en excès

$$
\Delta \sigma=k \cdot \Delta N_{0} \exp \left(-\frac{W_{1}}{k T}\right)
$$

le nombre de centres $\mathrm{D}^{0}$ en excès étant régi par un équilibre entre la génération et la recombinaison

$$
\frac{\mathrm{d}\left(\Delta N_{0}\right)}{\mathrm{d} t}=g-b\left(\Delta N_{0}^{2}+2 N_{0} \cdot \Delta N_{0}\right) .
$$

Sous éclairement constant et dans l'hypothèse $\Delta N_{0} \gg N_{0}$ (recombinaison bimoléculaire)

$$
\Delta N_{0}=\sqrt{g / b} .
$$

La loi en $F^{1 / 2}$ (la génération $g$ est proportionnelle à l'intensité lumineuse $F$ ) est souvent vérifiée [5]. La constante de recombinaison $b$ déduite de nos résultats expérimentaux [6] est en accord avec les valeurs trouvées dans la littérature [7]. Un calcul de cette cons- tante peut être fait dans l'hypothèse d'une distribution au hasard des paires de centres [8]

$$
b=2 \pi \omega_{0} \mathrm{e}^{-V / k T} / \alpha^{3}
$$

$\omega_{0}$ fréquence de phonons,

$V$ énergie d'activation associée à la réaction $2 \mathrm{D}^{0} \rightarrow \mathrm{D}^{+}+\mathrm{D}^{-}$,

$\alpha$ rayon de la fonction d'onde électronique du centre $\mathrm{D}^{0}$.

Par contre, dans l'hypothèse de paires proches voisines, ce calcul n'est pas effectué.

4. Fatigue photostructurale. - 4.1 FAITS EXPÉRIMENTAUX. - Dans cette étude les couches sont soumises à des cycles : une heure éclairement, une heure obscurité, pour diverses températures. La longueur d'onde utilisée $(\lambda=4250 \AA)$ est fortement absorbée $(99 \%$ des photons sont absorbés sur une épaisseur de l'ordre de $2000 \AA$ ). On observe une diminution du photocourant d'équilibre sous l'influence d'un éclairement prolongé [9].

Pour les températures élevées (par exemple $T=293 \mathrm{~K}$ ), le photocourant se stabilise en moins d'une heure (Fig. 2). Les cycles successifs sont identiques.

Pour les basses températures $(T=233 \mathrm{~K})$, la stabilisation est tout à fait différente (Fig. 3) :

- la décroissance est plus lente (durée de stabilisation $\sim 10 \mathrm{~h}$ ),

- au début d'une période d'éclairement, le photocourant reprend la valeur qu'il avait à la fin de l'éclairement précédent (les cycles successifs ne sont pas identiques).

Enfin remarquons que sur une couche régénérée par un passage à $T_{\mathrm{g}}$ et éclairée à $233 \mathrm{~K}$, on observe un comportement du même type mais avec un photo- 


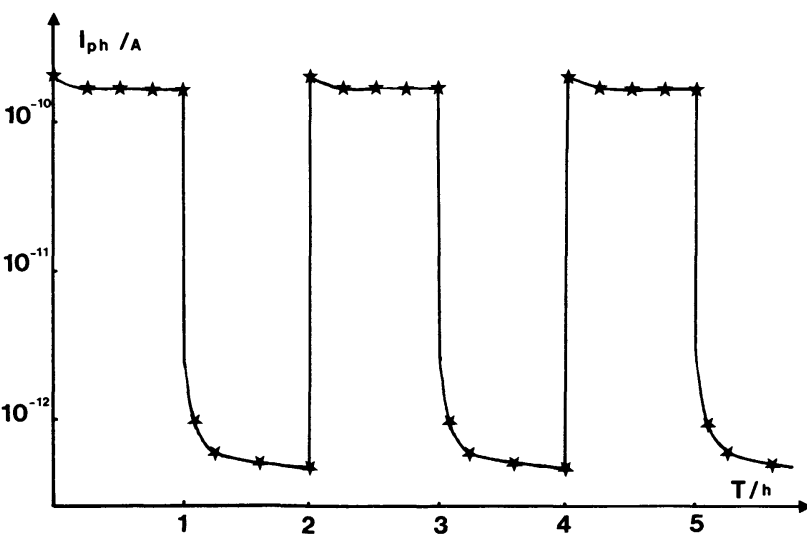

Fig. 2. - Variation du photocourant en fonction du temps (cycles $1 \mathrm{~h}$ éclairement, $1 \mathrm{~h}$ obscurité) à $T=293 \mathrm{~K}$.

[Photocurrent variation vs. time, (1 h illumination, $1 \mathrm{~h}$ obscurity), at $T=293 \mathrm{~K}$.]

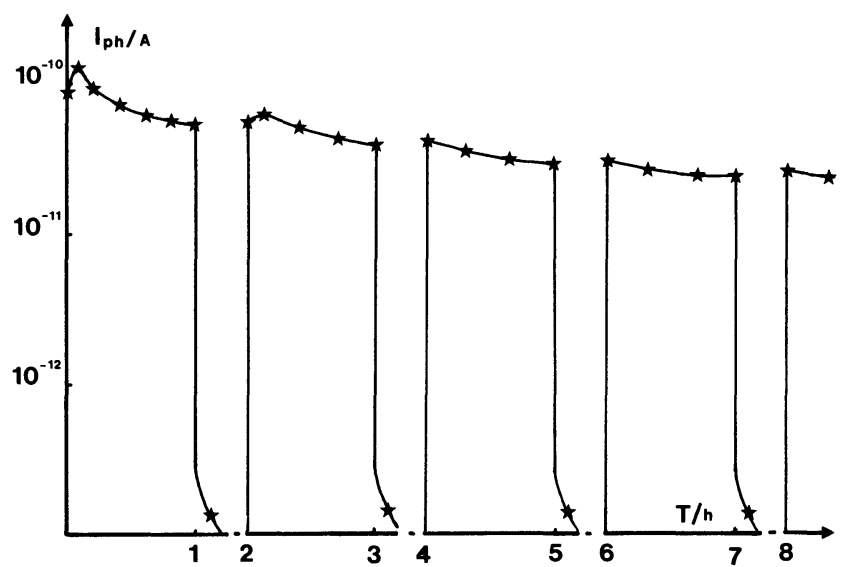

Fig. 3. - Variation du photocourant en fonction du temps à $T=233 \mathrm{~K}$ (couche non régénérée)

[Photocurrent variation vs. time at $T=233 \mathrm{~K}$ (non regenerated film).]

courant initial reproductible nettement plus élevé. La valeur stabilisée finale est la même.

4.2 INTERPRÉTATION. - A la mise sous éclairement le régime transitoire de photoconduction conduit très vite à un régime de quasi-équilibre (les constantes de temps du régime transitoire $\tau=\sqrt{g b}$ sont de l'ordre de $10^{-6} \mathrm{~s}$ [6]). L'évolution du photocourant observée $\left(\tau \simeq 10^{3}\right.$ à $\left.10^{4} \mathrm{~s}\right)$ ne peut donc s'expliquer par un régime transitoire.

A l'équilibre, le photocourant est de la forme :

$$
I_{\mathrm{ph}}=\mathrm{Cte} \cdot \sqrt{\frac{g}{b}} \exp \left(-\frac{W_{1}}{k T}\right) .
$$

Il est activé thermiquement [10] et proportionnel à $F^{1 / 2}$. A température constante $I_{\mathrm{ph}}$ est constant si $g$ et $b$ sont constants. En fait sous l'influence d'un éclairement prolongé, les centres de défauts se déplacent dans le matériau (déplacement des liaisons lors des échanges électroniques [11]) et la modification de la répartition spatiale des défauts conduit à une augmentation de la constante de recombinaison $b$.

Lors des périodes d'obscurité (les $\mathrm{D}^{0}$ se recombinent et le photocourant décroît), deux situations sont à envisager :

- si $T$ est élevé $(T=293 \mathrm{~K})$, les centres de défauts $\mathrm{D}^{+}, \mathrm{D}^{-}$, sous l'influence de l'agitation thermique reprennent une distribution au hasard. Lors d'un nouvel éclairement, on retrouvera la même constante $b$ qu'au début de l'éclairement précédent et donc le même photocourant,

- si $T$ est très inférieur à $T_{\mathrm{g}}(T=233 \mathrm{~K})$, les paires de centres ne peuvent reprendre une distribution au hasard : ils sont "gelés " et lors d'un nouvel éclairement la constante $b$ a gardé la même valeur qu'à la fin de l'éclairement précédent.

Une régénération de la couche par un passage à $T_{\mathrm{g}}$ conduit à une redistribution au hasard rapide, complète et reproductible des centres de défauts.

5. Saturation par forte absorption. -5.1 FAITS EXPÉRIMENTAUX. - L'étude des spectres de photoconduction à température $T=233 \mathrm{~K}$, à raison d'une heure d'éclairement par longueur d'onde entre 2000 et $10000 \AA$ est résumée sur la figure 4 . Trois spectres ont été enregistrés :

1) sur une couche préalablement saturée par un éclairement prolongé $(12 \mathrm{~h})$ avec des photons absorbés dans toute l'épaisseur de la couche $(\lambda=5250 \AA)$,

2) sur une couche régénérée par un passage à $T_{\mathrm{g}}$ balayée dans le sens des énergies croissantes,

3) sur une couche régénérée balayée dans le sens des énergies décroissantes.

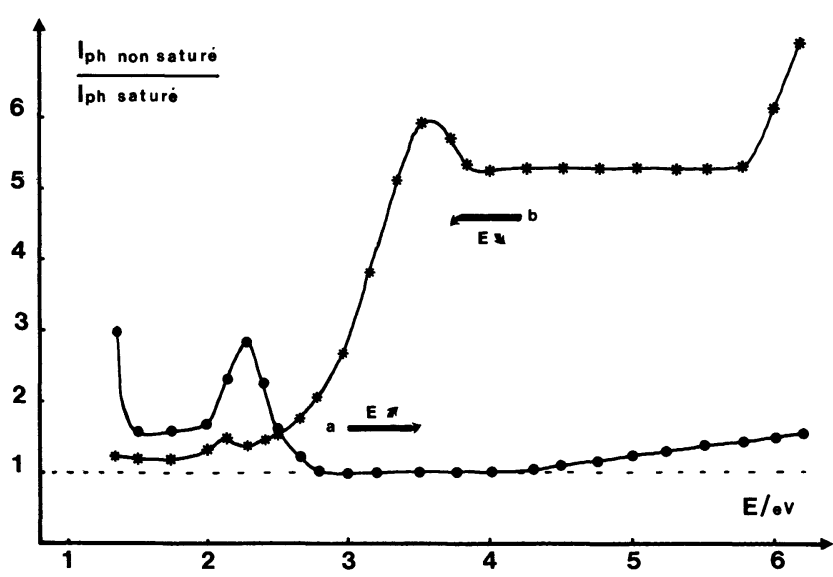

Fig. 4. - Comparaison des photocourants mesurés sur une couche régénérée à $T_{\mathrm{g}}$ et sur une couche saturée en fonction de $\lambda$ à $T=233 \mathrm{~K} . a$ ) Le spectre de la couche régénérée est effectué par énergies croissantes. $b$ ) Le spectre de la couche régénérée est effectué par énergies décroissantes.

[Comparison between photocurrents measured on a film regenerated at $T_{\mathrm{g}}$ and one saturated, $v$ s. $\lambda$ at $T=233 \mathrm{~K}$. a) Regenerated film spectrum is made with increasing energies. b) Regenerated film spectrum is made with decreasing energies.] 
Les courbes de la figure 4 représentent le rapport entre les photocourants obtenus sur une couche régénérée et la couche saturée en fonction de la longueur d'onde :

a) pour le spectre en énergies croissantes,

b) pour le spectre en énergies décroissantes.

5.2 INTERPRÉTATION. - La répartition spatiale des paires de défauts fournit une explication à l'ensemble des observations : sur la couche saturée, la répartition des paires comporte une forte proportion de paires proches et une constante $b$ élevée; sur les couches régénérées, en début de spectre, les centres sont distribués au hasard.

Lors de l'éclairement en énergies croissantes (courbe $a$ ), les photons sont d'abord faiblement absorbés, mais ils le sont dans toute l'épaisseur du matériau. Le photocourant de la couche régénérée, supérieur à celui de la couche saturée (rapport $>3$ ), tend progressivement vers la même valeur, la saturation s'achevant vers $\lambda=4750 \AA$.

Lors de l'éclairement en énergies décroissantes (courbe $b$ ), le photocourant de la couche régénérée est très supérieur à celui de la couche saturée (rapport $>6$ ) et le reste pour toutes les grandes énergies : les photons sont absorbés en surface et ne peuvent modifier la répartition spatiale des défauts en profondeur. Pour des énergies plus faibles $(\lambda \simeq 4000 \AA)$, l'absorption se fait de plus en plus profondément modifiant ainsi la répartition spatiale. Le rapport des photocourants tend vers l'unité.

La figure 5 donne l'évolution des stabilisations pour trois longueurs d'onde dans le cas du spectre (3) :

$\lambda=2300 \AA(99 \%$ des photons absorbés dans une épaisseur de $480 \AA$ ) pas de modification en profondeur,

$\lambda=4250 \AA(99 \%$ des photons absorbés dans une épaisseur de $2000 \AA$ ) saturation progressive

$\lambda=6000 \AA(69 \%$ des photons absorbés dans la couche) la couche est entièrement saturée.
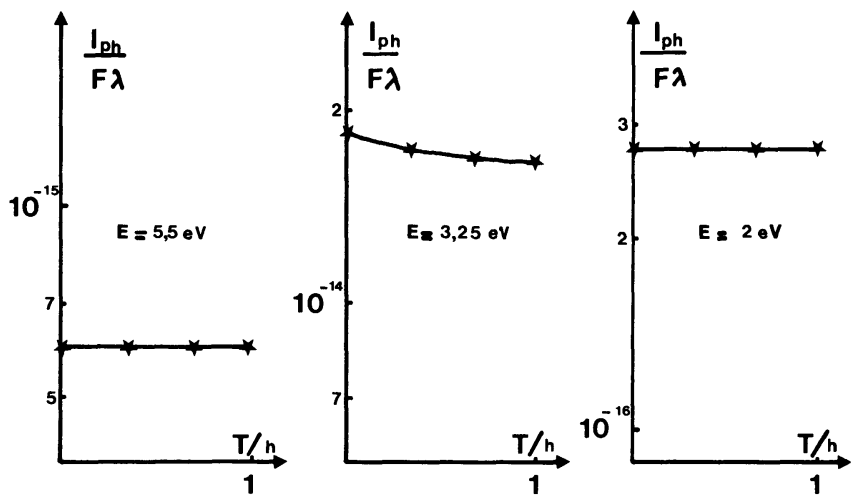

Fig. 5. - Stabilisations sur couche régénérée pour 3 énergies de photons.

[Stabilizations of regenerated film for different photon energies.]
6. Extinction optique. - 6.1 FAITS EXPÉRIMENTAUX. - Dans des travaux antérieurs [12], nous avons étudié les variations du photocourant dues à une radiation fortement absorbée $(\lambda=4700 \AA)$, lors de la superposition d'une radiation secondaire rouge ou infrarouge.

La figure 6 donne un exemple de cette évolution $\mathrm{au}$ cours du temps, à température ambiante $(T=290 \mathrm{~K}): I_{\text {bias }}$ est le photocourant dû à l'éclairement primaire. Ensuite on éclaire pendant une heure avec une radiation rouge puis on éteint.

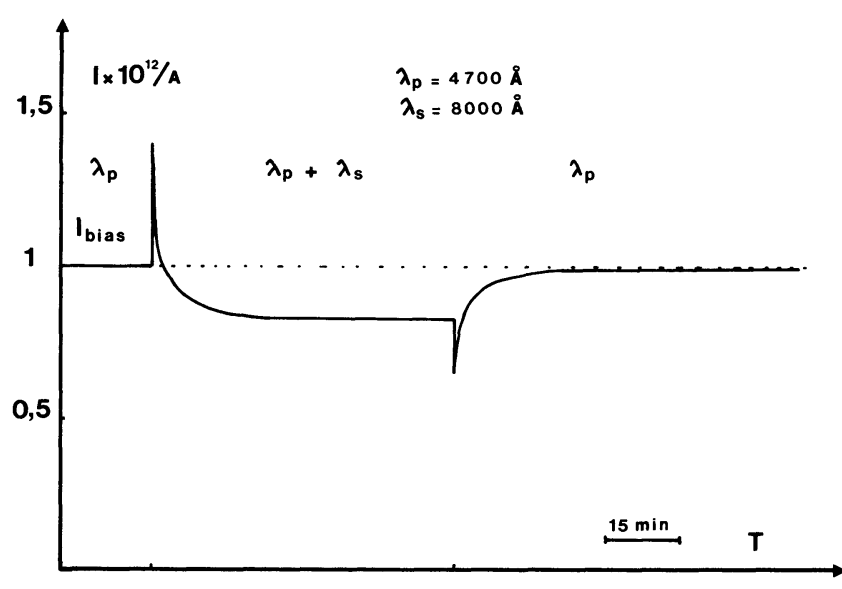

Fig. 6. - Action d'un éclairement secondaire (rouge) sur le photocourant créé par une radiation primaire (bleu).

[Secondary illumination effect (red) on photocurrent generated by primary illumination (blue).]

6.2 INTERPRÉTATION. - Une explication due à Rose [13] fait intervenir un trajet du trou (porteur de charge) à travers une distribution continue de pièges dans la bande interdite du matériau. Le modèle des défauts chargés fournit une explication intéressante.

Lors de l'éclairement par une radiation secondaire, on observe d'abord une excitation des nouveaux porteurs dus à l'augmentation des centres $D^{0}$. Ensuite cette radiation favorise les échanges électroniques entre niveaux profonds dans la bande interdite, c'est-àdire entre centres $\mathrm{D}^{0}$, modifiant de façon sensible la répartition des paires de défauts, d'où une augmentation de la constante $b$ et une diminution du photocourant qui peut devenir inférieur à celui créé par la seule radiation primaire $\left(I_{\text {bias }}\right)$.

Lors de l'extinction de la radiation secondaire, la suppression d'une partie de la génération et la constante $b$ élevée conduisent à une forte diminution du photocourant qui reprend sa valeur d'équilibre après un réarrangement de la distribution des centres de défauts.

7. Conclusion. - Le modèle des défauts chargés invoqué dans l'explication d'un certain nombre de phénomènes observés dans les chalcogènes (par exemple, fatigue de photoluminescence, absence de signal 
E.S.R...) a permis d'expliquer un certain nombre d'observations expérimentales et d'orienter les processus expérimentaux pour obtenir des résultats reproductibles en photoconduction.

La difficulté actuelle réside dans l'impossibilité de faire un calcul de la constante de recombinaison $b$ autrement que dans l'hypothèse d'une distribution de paires de défauts au hasard. Pour mener à bien un calcul, il serait nécessaire de connaître les paramètres intervenant dans le déplacement des liaisons.
L'étude qualitative a permis de remarquer qu'il existe deux situations extrêmes parfaitement reproductibles :

— état "régénéré » du matériau photoconducteur après recuit à la température de transition vitreuse,

- état « saturé » du matériau, obtenu par un éclairement prolongé avec une radiation absorbée en profondeur. A basse température cette situation sera métastable.

\section{Bibliographie}

[1] Mott, N. F., Davis, E. A., Street, R. A., Philos. Mag. 32 (1975) 961.

[2] Kastner, M., Fritzsche, H., Philos. Mag. 37 (1978) 199.

[3] Simmons, J. G., Taylor, G. W., J. Phys. C 7 (1974) 3051.

[4] Street, R. A., Solid State Commun. 24 (1977) 363.

[5] Fischer, R., Heim, U., Stern, F., Weiser, K., Phys. Rev. Lett. 26 (1971) 1182.

[6] Viger, C., Vautier, C., Carles, D., Phys. Status Solidi 44 (1977) 97.

[7] Rehm, W., Engemann, D., Fischer, R., Stuke, J., Proceedings of the thirteenth Int. Conf. on the Physics of Semiconductors, Rome (1976) 526.
[8] Street, R. A., Phys. Rev. 17 (1978) 3984

[9] Fleury, G., Viger, C., VAutier, C., soumis à Thin Solid Films.

[10] Viger, C., Vautier, C., Grenet, J., Revue Phys. Appl. 12 (1977) 767.

[11] MotT, N. F., J. Phys. 13 (1980) 5433.

[12] IKovic, I., Viger, C., VAUTier, C., 5th Int. Conf. on Amorphous and Liquid semiconductors, Garmisch-Partenkirchen (1973) 630.

[13] Rose, A., Concepts in photoconductivity and allied problems (Interscience Publisher, New York) 1963. 